\title{
6
}

\section{SUCCESSION PLANNING FOR THE BOARD}

\author{
Ray Crofts
}

For many boards, succession planning is a reactive activity, triggered by the imminent departure of an existing member, usually through retirement or exhaustion of tenure. For developed organisations it is the role of the nominations committee to forecast upcoming departures, anticipate what may be required in a replacement - and source the individual who would be that replacement.

However, as many businesses seek to become more agile and find that the pace of change is accelerating, waiting for a board member to retire or exhaust their tenure can result in stale boards which lack diversity of thought. The most efficient boards engage in a continual process of assessment and review to ensure their make-up is best suited to meet the upcoming strategic challenges of the business. There are a number of questions a board should be asking itself regularly:

- What is our purpose as a board? What do we need to fulfil that purpose?

- What are the reasons behind our current size and formulation?

- When was the last time we brought a new member to the board? 
- How do we classify the skills and attributes we need, and how often do we assess how well we fulfil those categories?

\section{Diversity of thought}

The most effective boards embrace diversity of thought by ensuring they contain a blend of skills, experience, and attributes. It is vital to ensure industry expertise, but also boards can benefit from external perspectives. Boards should continually challenge their assumptions, and a board which has been static for some time will often fall prey to groupthink. External assessment can play a crucial role in ensuring the board has the right blend of personalities and culture, as well as the requisite experience and knowledge to lead a business forward.

- Do we regularly challenge our decisions and assumptions?

- How do we demonstrate diversity of thought?

- What are our backgrounds? How are they similar/different?

- What unique skill/attribute do we each bring to the table?

- Do we actively seek opinions/advice from outside of our industry?

- What are the skills and knowledge that the organisation will need in the future that are currently under-represented on the board?

- Are we over-represented in certain areas/too dependent on certain skill sets?

- How well do we fulfil our obligations regarding length of tenure on boards? Why is this?

For board succession planning to be effective, it must be acknowledged as a priority by the whole board, which should be able to have regular, if difficult, conversations about how the board make-up aligns with the business strategy. However, it is also vital that overall ownership remain with the majority independent nominations committee, as it is their role to remain objective. Although stakeholders like the CEO are included in the process, no one individual is given the power of veto, as this can result in boards of like-minded individuals rather than a more diverse board. Well-managed succession planning should include as wide a range of stakeholders as possible. For example, offering shareholders visibility of a structured and planned process has been known to diffuse tensions between board and 
shareholders, heading off the threat of activism on the part of frustrated shareholders, who often cite "ineffective boards" or "poor board composition" when raising challenges or seeking better representation.

- How do we demonstrate transparency in our process of recruitment to the board?

- Who has ownership of the succession planning process? How does this manifest?

- How do we communicate our plans for future recruitment on the board to other stakeholders?

\section{Effectiveness reviews}

Board effectiveness reviews can be carried out internally or via an external consultant. These regular assessments give a board a framework to think about its own make-up and consider making changes. If changes are required, they also offer a basis to consider where the gaps in knowledge and skills lie, enabling the board to construct a candidate profile which covers not just experience and skills but also style and the all-important cultural fit.

- How frequently do we review our effectiveness as a team?

- What improvements have we made as a result of an effectiveness review?

- How do we collectively demonstrate our commitment to the review process?

- What key elements define our culture? How are these demonstrated?

\section{Role specifications}

When building a specification, it is important to be pragmatic, and understand which elements are essential and which are not. It is also an opportunity to take a fresh look at previous restrictions on specifications and challenge these. Some boards for example prefer not to take on first-time non-executive directors, but these individuals are often younger, with a valuable, forward-looking skill set. Or you may consider looking outside of your own industry. Increasingly, boards are looking to "future proof" 
with a broader range of skills and experiences, and seeking directors with experience in sectors like tech, marketing, human resources, and even sustainability. Some businesses even seek to have a representative of employees on the board, to offer a different perspective. Diversity amongst the board - be it in skill set, experience, gender, age or race - should be actively encouraged.

- In what way will the business develop over the next five years, and what skills and attributes will the board need to support that development?

- What assumptions are we making about suitable/unsuitable candidates? Where do these come from?

- Have we considered a workers' representative on the board?

- What associated industries could provide alternative candidates with a fresh perspective?

\section{Recruitment process}

An objective and transparent recruitment process serves to reassure stakeholders and governing bodies. Many boards choose to use an external supplier when recruiting new directors for this reason. It is vital that any internal candidates proposed for a role be included in the same process, to be benchmarked against the same criteria to ensure transparency and objectivity.

- How can we demonstrate that our process is objective?

- Whom do we allow visibility of the recruitment process? Why?

- When we recruit, what kind of restrictions do we place on the recruitment process? Are these necessary?

- Do we ensure that our criteria are balanced and accurately reflect our needs? How do we benchmark against these?

To close the circle, the process must be subject to constant review and evaluation. Only by considering the success of the appointment, the current strength of the board, and its fitness to lead the future strategy of the business will you ensure the board remains ready to tackle the challenges of tomorrow. 
- Looking back, how well do we anticipate individual departures from the board?

- How successful is our appointment process?

- Why have previous appointments been successful/unsuccessful? 